\title{
Understanding the Anomalous Short-Range Spatial Correlation Of Fe and Sn in Neutron- Irradiated Zr Alloys
}

Benjamin Jenkins ${ }^{1}$, Chris Grovenor ${ }^{2}$ and Michael Moody ${ }^{3}$

${ }^{1}$ University of Oxford, OXFORD, England, United Kingdom, ${ }^{2}$ Oxford University, OXFORD, England, United Kingdom, ${ }^{3}$ University of Oxford, Oxford, England, United Kingdom

Zirconium alloys are extensively used in light water nuclear reactors. However, deleterious changes in their properties during operation limit the safe operational lifetime of the components, and fuel rods must be regularly replaced [1]. There is an associated economic and environmental cost to this, and the ability to manufacture Zr-based alloys capable of operating for longer times in extreme environments is highly desirable. Designing alloys that are more robust in the future relies on gaining an understanding of the mechanisms that cause $\mathrm{Zr}$ alloys to undergo these changes, many of them at the nanoscale. In order to gain a fundamental understanding of these phenomena, it is necessary to complement modelling studies with high-resolution characterisation. Atom probe tomography (APT) is a 3-dimensional characterisation technique with a unique combination of high spatial and chemical resolution, and has previously been used to gain insight as to the nanostructural development of $\mathrm{Zr}$ alloys exposed to irradiation [2,3]. In this study, as part of the international MIDAS project, a series of Zr-based alloys were neutron-irradiated to a variety of fluences in the BOR-60 reactor. The nanostructure of each alloy has been characterised using APT after exposure to several damage levels $(4.4,17$, and $33 \mathrm{dpa}$, respectively). This presentation will focus on how the distribution of solute atoms throughout the specimens changes during exposure to irradiation - in particular, a focus will be on the relationship between $\mathrm{Sn}$ and Fe distributions. Previous STEM/EDX work on Zircaloy-2 has demonstrated that $\mathrm{Sn}$ and Fe atoms are anti-segregated on the scale of approximately tens of $\mathrm{nm}$ [4]. This behaviour was again confirmed in the APT study of these neutronirradiated alloy. However, notably at finer length-scales of 1-3 nm spatial-correlation in the distribution of $\mathrm{Sn}$ and $\mathrm{Fe}$ was also simultaneously observed (Figure 1 (b)). This presentation will focus on this seemingly contradictory observation of $\mathrm{Fe}-\mathrm{Sn}$ being anti-segregated but co-clustered in irradiated Zircaloy-2, and an explanation for the observed behaviour will be proposed. The spatial relationship between solute elements in other neutron-irradiated Zr-based alloys (e.g. ZIRLO) has also been characterised using APT and these results will be compared to data from TEM studies [5]. The relationship between the nano-structural evolution and changes in the macroscopic properties of the alloys will be also discussed, alongside how this information can be used to validate or improve models within the community. The research used UKAEA's Materials Research Facility, which has been funded by and is part of the UK's National Nuclear User Facility and Henry Royce Institute for Advanced Materials. 
(a)

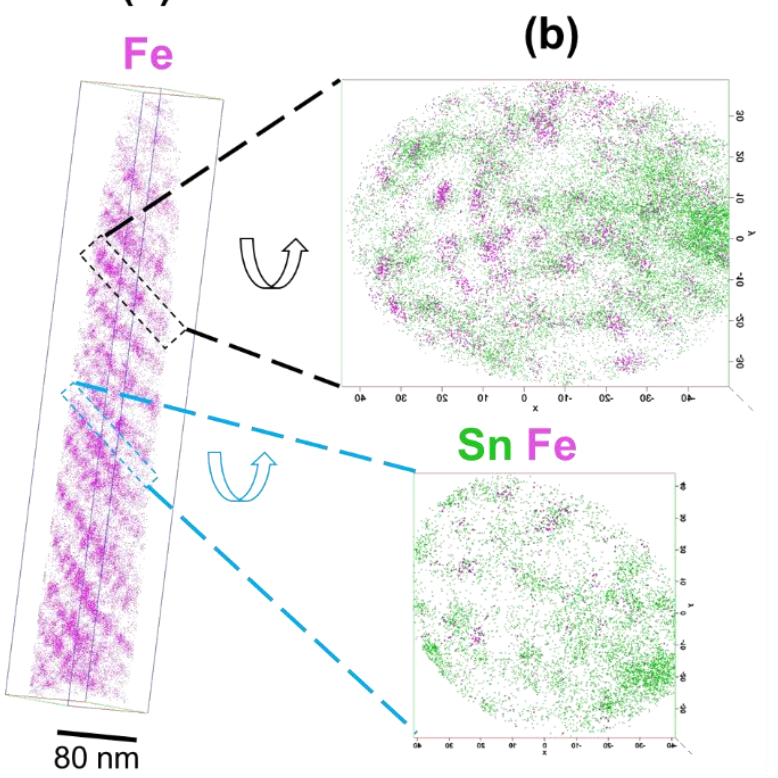

(b)

(c)

(d)

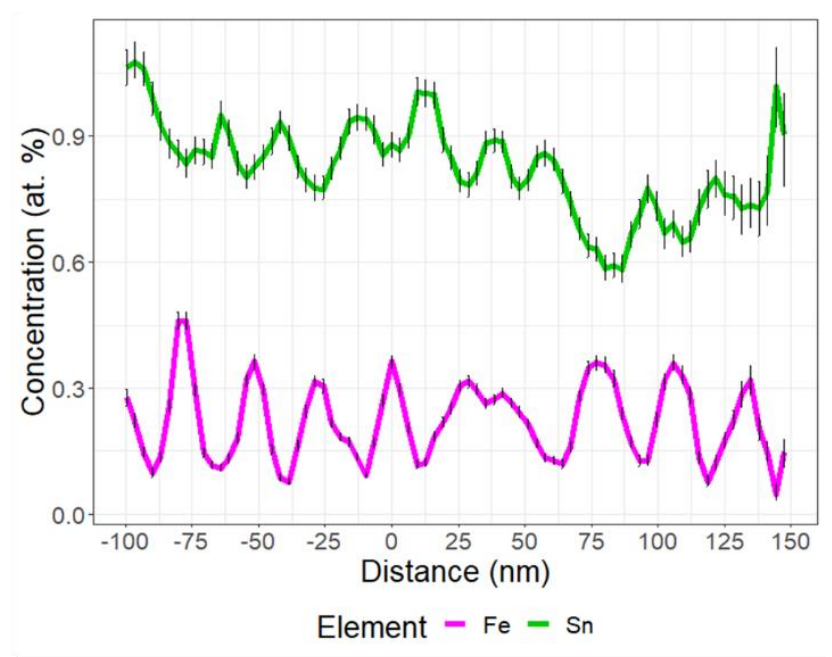

Figure 1. Atom maps showing the distribution of (a) Fe atoms in a Zircaloy-2 sample irradiated to 4.4 dpa. Atom maps show the distribution of $\mathrm{Sn}$ and $\mathrm{Fe}$ atoms in (b) the plane of a dislocation array, (c) between the dislocation array. (d) Is a one-dimensional concentration profile take perpendicularly to the dislocation arrays in (a).

\section{References}

[1] S.J. Zinkle, K.A. Terrani, J.C. Gehin, L.J. Ott, L.L. Snead, Accident tolerant fuels for LWRs: A perspective, J. Nucl. Mater. 448 (2014) 374-379. doi:10.1016/j.jnucmat.2013.12.005.

[2] G. Sundell, M. Thuvander, H.O. Andrén, Tin clustering and precipitation in the oxide during autoclave corrosion of Zircaloy-2, J. Nucl. Mater. 456 (2015) 409-414. doi:10.1016/j.jnucmat.2014.10.003.

[3] G. Sundell, M. Thuvander, P. Tejland, M. Dahlbäck, L. Hallstadius, H.O. Andrén, Redistribution of alloying elements in Zircaloy-2 after in-reactor exposure, J. Nucl. Mater. 454 (2014) 178-185. doi:10.1016/j.jnucmat.2014.07.072.

[4] A. Harte, D. Jädernäs, M. Topping, P. Frankel, C.P. Race, J. Romero, L. Hallstadius, E.C. Darby, M. Preuss, The effect of matrix chemistry on dislocation evolution in an irradiated $\mathrm{Zr}$ alloy, Acta Mater. 130 (2017) 69-82. doi:10.1016/j.actamat.2017.03.024.

[5] G. He, J. Liu, K. Li, J. Hu, A.H. Mir, S. Lozano-Perez, C. Grovenor, Investigating the stability of second phase particles in Zr-Nb alloys under irradiation, J. Nucl. Mater. 526 (2019) 151738. doi:10.1016/j.jnucmat.2019.151738. 